\title{
LYMPHOSCINTIGRAPHIC EVALUATION OF PARACOCCIDIOIDOMYCOSIS PATIENTS
}

THESIS: B. L. Griva submitted this thesis for her Doctorate in Tropical Diseases at Botucatu School of Medicine, São Paulo State University (UNESP), Botucatu, São Paulo, Brazil, 1999.

Advisor: Professor Rinaldo Poncio Mendes

ABSTRACT: Lymph node involvement is very frequent in paracoccidioidomycosis (PBM) mainly in its juvenile form. PBM also affects the lymphatic system of patients with the chronic form as it was confirmed by bipedal lymphangiography and autopsy. Lymphoscintigraphy has been used to evaluate lymphedema and cancer. The purpose of this investigation was to study the lymphoscintigraphic variables in a critical manner, to functionally evaluate the lymphatic system of patients with different forms of PBM, and to evaluate the influence of the antifungal treatment on the lymphoscintigraphic variables by lymphoscintigraphy of the extremities. Forty-six patients with PBM were studied. Twenty-one had the juvenile and 25 the chronic form. Patients with the juvenile form were divided again into three subgroups according to age. Eleven healthy volunteers were evaluated as controls. All of them were injected with $37 \mathrm{MBq}$ of dextran $500-{ }^{99 \mathrm{~m}} \mathrm{Tc}$ into the first interdigital space of each foot, and blood was obtained for serum albumin determination. Twenty-nine patients were evaluated after the beginning of the antifungal treatment. The time elapsed between both exams was smaller in patients with the juvenile form than in those with the chronic form. Semi-quantitative variables, such as the intensity of visualization of the radiotracer and the pattern of the time-activity curve with regions of interest over ilioinguinal lymph nodes and legs, were studied. Quantitative variables were also evaluated including the velocity of visualization of the lymph nodes, lymph flow ratio, and lymph node radiotracer uptake. The error propagation was calculated to assess the lymph flow ratio accuracy. Correlation between variables and serum albumin was 
done. The intensity and velocity of visualization of the radiotracer, the lymph flow ratio, and the pattern of the time-activity curve were useful variables to the evaluation of patients with the juvenile and the chronic form of PBM. The lymph flow ratio calculated by the division of both areas under the time-activity curve was the most accurate compared to the others calculated. The lymphoscintigraphic evaluation of patients before the institution of the antifungal treatment showed an enhanced pattern of lymph flow in patients with the juvenile form of PBM not related to age. This finding is probably related to the low level of serum albumin found in these patients. The antifungal treatment did not influence all but one variable studied in patients with the juvenile form of PBM. The radiotracer progression was smaller after treatment in both groups. The intensity and velocity of visualization of the radiotracer were higher after treatment in patients with the chronic form of PBM. These results showed that in spite of the later lymphoscintigraphic follow-up, the antifungal treatment had a stronger influence on the functional status of the lymphatic system of patients with the chronic form than on those with the juvenile form of PBM.

KEY WORDS: paracoccidioidomycosis, lymphatic system, lymphoscintigraphy; dextran-technetium 99m.

CORRESPONDENCE TO: B. L. GRIVA, Departamento de Doenças Tropicais e Diagnóstico por Imagem, Faculdade de Medicina de Botucatu, UNESP, Distrito de Rubião Junior, s/n, 18618-000, Botucatu, SP, Brasil. Phone: 551438116212. Email: bgriva@fmb.unesp.br. 